\title{
Methodology optimizing SAGE library tag-to-gene mapping: application to Leishmania
}

\author{
Sondos Smandi, Fatma Z Guerfali, Mohamed Farhat, Khadija Ben-Aissa, Dhafer Laouini, Lamia Guizani-Tabbane, \\ Koussay Dellagi and Alia Benkahla*
}

\begin{abstract}
Background: Leishmaniasis are widespread parasitic-diseases with an urgent need for more active and less toxic drugs and for effective vaccines. Understanding the biology of the parasite especially in the context of host parasite interaction is a crucial step towards such improvements in therapy and control. Several experimental approaches including SAGE (Serial analysis of gene expression) have been developed in order to investigate the parasite transcriptome organisation and plasticity. Usual SAGE tag-to-gene mapping techniques are inadequate because almost all tags are normally located in the 3'-UTR outside the CDS, whereas most information available for Leishmania transcripts is restricted to the CDS predictions. The aim of this work is to optimize a SAGE libraries tagto-gene mapping technique and to show how this development improves the understanding of Leishmania transcriptome.
\end{abstract}

Findings: The in silico method implemented herein was based on mapping the tags to Leishmania genome using BLAST then mapping the tags to their gene using a data-driven probability distribution. This optimized tag-to-gene mappings improved the knowledge of Leishmania genome structure and transcription. It allowed analyzing the expression of a maximal number of Leishmania genes, the delimitation of the $3^{\prime}$ UTR of 478 genes and the identification of biological processes that are differentially modulated during the promastigote to amastigote differentiation.

Conclusion: The developed method optimizes the assignment of SAGE tags in trypanosomatidae genomes as well as in any genome having polycistronic transcription and small intergenic regions.

\section{Background}

Leishmania, the causative agent of leishmaniasis, is a protozoan parasite of the order Kinetoplastida. The Leishmania major genome is $33 \mathrm{Mb}$ in size with a karyotype of 36 chromosomes. There are 911 RNA genes, 39 pseudogenes, 8272 protein coding genes of which $36 \%$ can be ascribed a putative function. The means of CDS and intergenic regions length are $1901 \mathrm{bp}$ and 2045 bp, respectively [1].

Leishmania species exist in two distinct stages within the mammalian host they infect. Promastigotes, present in the sand fly insect vector, are inoculated to mammalian hosts, where they transform into amastigotes, a

\footnotetext{
* Correspondence: alia.benkahla@pasteur.rns.tn

Laboratoire d'Immuno-Pathologie, Vaccinologie et Génétique Moléculaire (LIVGM), WHO Collaborating Center for Research and Training in

Leishmaniasis, Institut Pasteur de Tunis, 13 place Pasteur BP74 1002, Tunis, Tunisia
}

() 2012 Smandi et al; licensee BioMed Central Ltd. This is an Open Access article distributed under the terms of the Creative Commons Attribution License (http://creativecommons.org/licenses/by/2.0), which permits unrestricted use, distribution, and reproduction in any medium, provided the original work is properly cited. form adapted to survive within these mammalian host cells. The molecular events allowing the differentiation from promastigotes to amastigotes are still poorly understood. The post-transcriptional and/or post-translational regulation of genes involved in several biological processes is certainly important to adapt the parasite to survive in the harsh conditions of the parasitophorous vacuole and to circumvent the host's immune response. Hence, a systematic identification of these genes is necessary to understand the mechanisms underlying parasite intracellular survival.

Several gene expression experiments were performed on different Leishmania species using DNA, cDNA, oligonucleotides microarrays or SAGE technology [2-8]. Different studies performed at the transcriptomic level have focused, either on genes differentially expressed between promastigote and amastigote stages, or between distinct Leishmania species. Modulated genes encode
C Biomed Central 
for proteins with either hypothetical or unknown functions or for proteins with known function e.g. surface proteins, kinases, maintenance protein, metabolic enzymes, structural genes, transporters, and heat shock proteins.

SAGE is an approach that allows the rapid, quantitative, simultaneous and detailed analysis of thousands of transcripts [9], and is a powerful tool for the analysis of genome-wide gene expression without requiring knowledge of the gene content [10]. It was successfully used in a wide variety of organisms and applications including the elucidation of diseases [11], the detection of transcripts expressed at low levels [12] and the discovery of new genes [13]. Its output is a list of short sequence tags which size depend on the specific SAGE technology used. One of the critical steps using SAGE technique is the tag-to-gene mapping. Classical methods involve the mapping of any SAGE tag to the 3' most tag within each transcript. These methods either use known 3'UTR or artificially extend the 3'UTR of predicted genes or both. Unfortunately, these methods are not applicable for the study of organisms like Leishmania for which the full transcripts and the approximate length of their 3'UTR are unknown.

In a previous report [3] using SAGE, we have provided a large-scale gene expression profile of Leishmania major (L. major) promastigotes $(\mathrm{Lm})$ and Leishmania infected Monocyte Derived Macrophages $(\mathrm{MDM}+\mathrm{Lm})$. The used SAGE technology produces 14 nt cDNA tags. The tag-to-gene assignment technique was basic and the assignment restricted to the most abundant tags and to tags preferentially expressed by intra-macrophagic parasites. As a result several tags/genes were excluded from the analysis because they could not be assigned to their respective genes or because they had a steady expression. Considering this limitation, we present here a novel method that optimizes the tag-to-gene mapping process for all expressed tags. This method does not validate the SAGE libraries, it exploits the genomic sequence and gives different confidence values to each of the hits of a SAGE-tag in the genome. The confidence values were defined according to parameters obtained through the kernel density estimation (kde) [14] of a distribution of unambiguously assigned tags. A detailed workflow is shown in Figure 1.

The implemented technique allowed the assignment of a maximum number of SAGE-tags, and the evaluation of the expression of their transcripts and the delimitation of some 3'UTR. These results pushed a step forwards the initial analysis [3] by integrating the expression of a larger number of genes and extending the knowledge of Leishmania 3'UTR. Additionally, we systematically characterized the function of genes

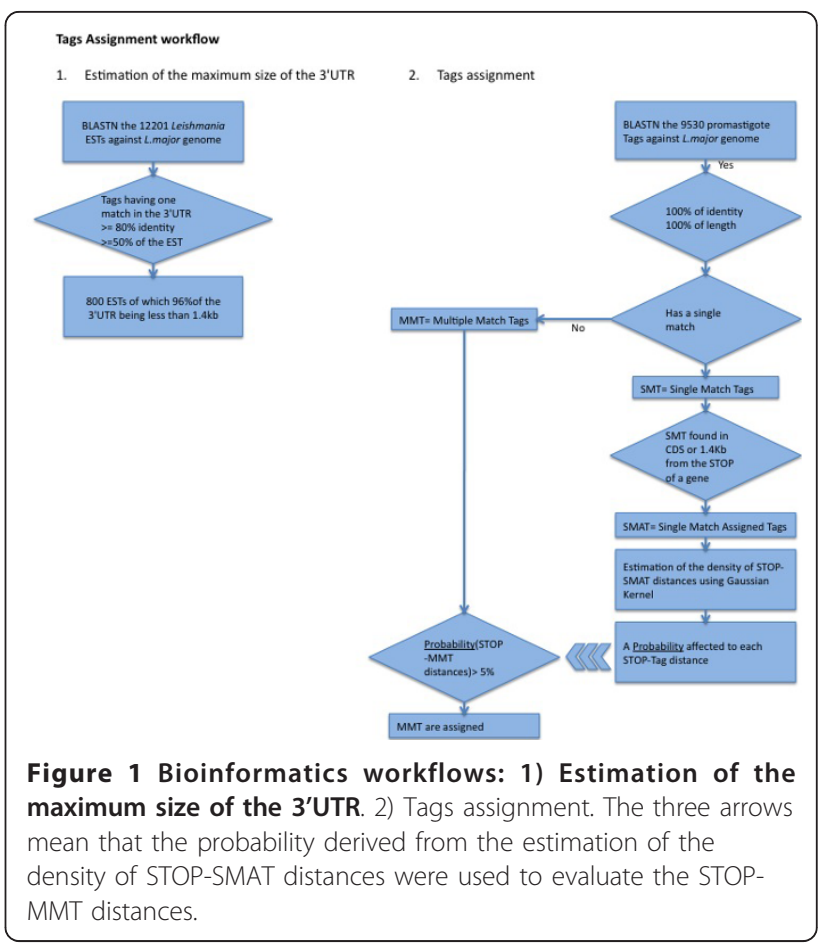

differentially expressed in Lm versus the library generated at amastigote stage $(\mathrm{MDM}+\mathrm{Lm})$ [3].

\section{Results}

The main result of the present study is the development of a tag-to-gene assignment tool ( $\mathrm{R}$ program available in the additional files). The developed program assigns a maximum number of SAGE-tags [3], to their respective genes. Multiple match tags (MMT) are the tags which assignment was the trickiest. The program uses a datadriven probability derived from the mapping of a set of single match assigned tags (SMAT), and evaluates the most probable tag-to-gene association of MMT. Results are available in Additional file 1. The limit $1.4 \mathrm{~kb}$, maximal tolerated distance between the CDS and the end of the 3'UTR, was derived from the 800 mapped 3'ESTs available in Genbank on November 2010 (See Figure 2 and Materials and Methods - Estimation of the maximum size of the 3'UTR paragraph). Because $1.4 \mathrm{~kb}$ might be considered as being too short or too large, the authors have parameterized this length into the source $\mathrm{R}$ program leaving to the user the freedom to rerun the program with the distance that suits them best.

According to the method implemented for tags assignment, 7766 out of the 9530 unique tags were mapped on the parasite genome. Among these, 4168 tags were classified as single match tags (SMT) and 3598 as MMT. Among SMTs, 3171 were classified as SMAT and assigned to 2538 different genes. The $3598 \mathrm{MMT}$ had 13617 mappings (on average 4 mappings/tag), and 


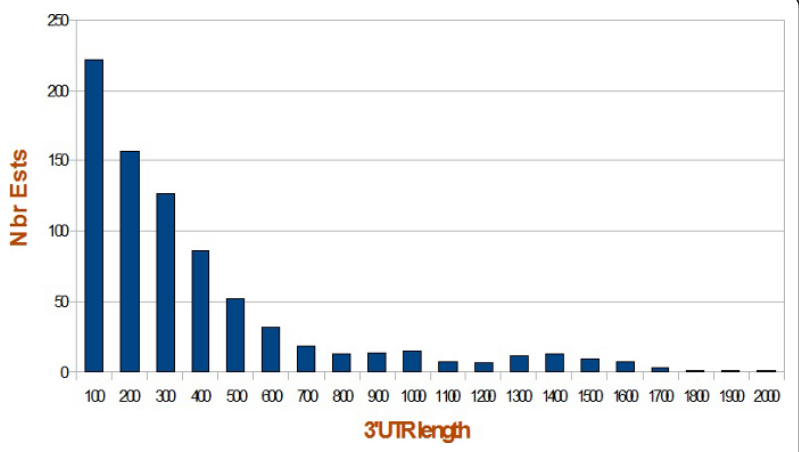

Figure 2 Histogram illustrating the size distribution of the 3'UTR of 800 3'ESTs mapped on the L. major genome. 573 ESTs overlapped with the CDS and with the 3'UTR of transcripts and 227 ESTs were in the $3^{\prime} U T R$ of transcripts. $96 \%$ of the latter $3^{\prime} U T R$ are less than $1.4 \mathrm{~kb}$.

out of these, 908 tags were assigned to 832 different genes. In total, the implemented strategy was able to successfully assign 4079 tags $(52.56 \%$ of total sequenced tags) to 3094 genes (37\% of total genes) (Additional file 2).

Because gene expression is known to be driven from the 'sense' and 'antisense' strand (complementary) of DNA in Leishmania [15-17], we have used our SAGE data to predict the antisense gene transcription. From the 3171 SMT, 2449 were assigned to genes in the same direction ('sense') and 722 to genes in the opposite direction ('antisense'). From the 908 assigned MMT, 610 were sense and 298 antisense. In total, 2148 genes showed no antisense transcription and 470 no sense transcription, whereas 476 showed both a sense and an antisense transcription. This result estimates the rate of antisense genes as being higher than expected ( $30 \%)$.

Among the 3094 identified genes, 772 were represented with different tags ( 275 with tags in the same direction, 21 with tags in the opposite direction and 476 with tags in both directions). 11 of these had significant expression changes (LmjF10.0090, LmjF15.0950, LmjF15.1203, LmjF24.2080, LmjF26.2220, LmjF29.1730, LmjF29.2370, LmjF34.2900, LmjF35.1890, LmjF36.3620 and LmjF36.6680); the direction of their expression change was the same according to the different tags counts. The list of these 11 genes is enriched in genes showing sense and antisense co-transcription (5/11) an observation that might reflect one of Leishmania posttranscriptional regulatory mechanisms.

Apart from the estimation of the expression of a maximum number of genes and the identification and the GO characterization of a maximum number of differentially expressed genes; the developed method allowed the delimitation of some 3'UTR.

\section{Functional characterization of differentially expressed} genes

A large proportion of $L$. major genes are differentially expressed between promastigote and amastigote stages [3]. Indeed, a total of 304 genes showed a significant differential expression between $\mathrm{Lm}$ and MDM + Lm, indicating that they might play a role in the parasite differentiation and/or in Leishmania virulence. Among these 304 genes, 189 and 115 genes were preferentially expressed by amastigotes and promastigotes respectively. The functional analysis of these genes using GOTermMapper and GOTermFinder revealed that 102 genes could be classified according to their biological processes, whereas 202 genes had no functional annotation (Additional file 3 and Additional file 4). KEGG pathways [18] associated to these genes (Additional file 4) revealed that 65 genes are Involved in known pathways. This result gives an idea about the Leishmania processes and pathways that are preferentially activated during the different stages.

\section{3'UTR characterization}

PRED-A-TERM allowed the prediction of 1581 3'UTR extremities (337 were located between the STOP and the tag; 452 were located between the tag and the following CATG; 648 between the following CATG and the start of the following gene; and 144 after the start of the following gene). Following the approach detailed in the paragraph 3'UTR characterization (in the Materials and Methods), we were able to validate the PRED-A-TERM predictions for 478 different L. major genes (Additional file 5): These were grouped into 452 predictions validated using the tags data and 26 using the EST data.

\section{Discussion}

To assign the maximum number of tags to their respective genes in a SAGE library constructed from metacyclic Leishmania promastigotes [3], we developed a method that evaluates the most likely tag-to-gene association of MMT. The implemented method allowed the assignment of a maximum number of SAGE tags, without arbitrarily fixing the size of the 3'UTR as it was previously done for the assignment of Arabidopsis tags $[19,20]$.

Unlike the method presented by Malig and colleagues [21], the one developed here dealt with experimental tags and provided an estimation of the confidence on a tag-to-gene assignment. Malig and colleagues method [21] dealt with virtual SAGE tags and evaluated the tagto-gene assignment intuitively. We also preferred to use what we have learned from the frequency distribution of the distance of SMAT from the STOP of the associated gene rather than extending the 3'UTR by a fixed 
number of nucleotides as in Pleasance and colleagues method [22].

SAGE technology is being routinely used to detect antisense transcription [23]. Such prediction has the same value as the prediction of sense transcription. The fact that antisense is being more common than expected $(\sim 30 \%)$ is presumed since gene regulation in Leishmania is driven through post-transcriptional mechanisms likely modulated through antisense transcription [24].

In our previous study [3], the purpose was to capture strong functional signals emitted during the differentiation from promastigotes to amastigotes. The tag-to-gene assignment was basic and not generalized to all tags. The assignment was restricted to the most abundant tags $(1.1 \%$ of the total number of unique tags) and to tags preferentially expressed by intra-macrophagic parasites. The optimization of the tag-to-gene assignment procedure herein allowed the identification of a maximum number of expressed and differentially expressed genes. The consequence of the latter allowed the identification of 76 additional genes preferentially expressed by intra-macrophagic amastigotes [3].

Several statistical tests are available to evaluate differential expression in SAGE libraries. The comparison of the output of the used test [25] to that of other tests (Student's test [26] and Shapiro-Wilk test [27]) showed, as expected, no significant differences. Therefore, the used statistics should not impact on the interpretation of the results. However, it has to be noted that libraries of a size lower than 120000 (the size recommended for SAGE experiments [28]), could under-estimate the number of expressed and differentially expressed genes which could impact on the interpretation of the results. This might explain why some genes are not observed as being constitutively expressed as previously reported [6].

Compared to our previous study, the functional GO characterization was done for all differentially expressed tags. GOTermMapper, GoTermFinder and KEGG pathways annotation indicated that the list of modulated genes is enriched in genes which end products are involved in RNA 'translation', inferring that translation related processes are affected by the intracellular development of the parasite. This result agreed with those reported by $\mathrm{McNi}$ coll and colleagues [8] who suggested that protein translation is affected during promastigote to amastigote differentiation (a weak correlation was observed between the transcriptome and the proteome levels at the amastigote stage and no correlation at the promastigote stage) and that translational and post-translational mechanisms are important for controlling gene expression.

Clayton and Shapira show in 2008 [29] that the parasite uses a polycistronic transcriptional approach and that mRNA abundance is regulated by post-transcriptional mechanisms driven through elements located in the 3' untranslated region (3'UTR) $[8,29]$. Therefore, to be properly interpreted, transcriptome data would need the development of tools that allow the study of the post-transcriptional regulatory elements located in the 3'UTR. The first step towards this development is a better delimitation of the 3'UTRs of known genes.

As the 478 3'UTR extremity predictions were supported by experimental data (i.e.: Leishmania 3'EST and/or $L$. major tags), they could be considered as true predictions and the predicted 3'UTR could be used, in our opinion, straight away for the investigation of post-transcriptional regulatory motifs. Additionally, the 3'UTR extremity predictions located between the CATG following a tag and the start of the following gene can be valid and would have to be validated through the sequencing of the corresponding transcript. Predictions located elsewhere should be considered as false positives unless they correspond to overlapping genes which existence is to be proven in Leishmania.

The presented method was not able to assign 5451 out of 9530 tags, among which 1764 were not mapped to $L$. major genome, which could be due to several reasons including tags sequencing errors (false positives), small size of the tags (14 nt), or polymorphic variations between the Friedlin strain used for the genome sequencing and the strain used to construct our SAGE library, or the presence of microbial component. Some unassigned tags could also belong to new protein or nonprotein coding genes, absent from the GeneDB catalogue. These tags would gain to be further investigated to obtain an exhaustive L. major genes catalogue. It has also long been known that non-coding RNAs are relatively common in trypanosomatids, and represent relatively stable processing products from polycistronic transcripts [30]. Since the existence of non-protein coding genes was not considered because of the absence of a dedicated catalogue, this has probably contributed to the raising of the mis- and non-assigned tags rate and implied that a proportion of the remaining non-assigned tags (the SMT mapping between two genes and some MMT) could belong to this class of genes. The lack of exhaustive knowledge about the actual full transcripts catalogue could also generate erroneous tag-to-gene associations of multiple match tags. Polymorphism within the $L$. major genus and the tags sequencing errors were additional parameters that can increase these rates.

The Leishmania genome was characterized by the presence of duplicated segments containing large gene families [1]. MMT mappings into members of the same gene family were difficult to assign and often remained unassigned. A complementary approach would be considering the assignment of these tags to conserved gene families' rather than individual genes. 


\section{Conclusions}

In the present study, we developed a novel approach for a better assignment of the SAGE tags to their genes for all organisms having polycistronic transcription and small intergenic regions. It expanded classical tag assignment methods to a method that does not require any extended knowledge concerning the 3'UTR. The implicit consequence of optimizing the assignment process was to approximate sense and antisense tag expression and to identify a maximum number of Leishmania genes that are differentially expressed. It should however worthy to note that all these assignments need to be validated experimentally.

The comparison of gene expression profile between promastigote and amastigote stage and the systematic GO classification of modulated proteins allowed the identification of the key biological processes that are modulated during differentiation from promastigotes to amastigotes. An exhaustive functional annotation of the genes involved in these processes would be helpful in understanding the mechanisms of intracellular parasite differentiation and in the identification of new drug target proteins.

In addition, a new approach indicating the most likely 3'UTR extremity of Leishmania genes is presented. This approach is based on the assessment of predicted Leishmania 3'UTR mRNA extremity by supporting biological evidences (Lm tags, Leishmania Expressed Sequence Tags and one prediction tool [31]).

\section{Materials and methods} Generation of SAGE libraries

SAGE libraries were previously generated by our group and corresponded to L. major GLC94 metacyclic extracellular promastigotes $(\mathrm{Lm})$ and to L. major GLC94 in their intracellular form within infected macrophages $(\mathrm{MDM}+\mathrm{Lm})$. All details about the parasite culture and preparation, the RNA isolation, and the SAGE library construction and its computer-based analysis were described by Guerfali and colleagues [3]. Lm was obtained from purified metacyclic L. major promastigotes and contained a total of 33,906 SAGE-tags corresponding to a non-redundant set of 9,530 different tags. $\mathrm{MDM}+\mathrm{Lm}$ was sequenced from $L$. major-infected macrophages and contains 57,514 tags corresponding to a non-redundant set of 24,418 tags. Both libraries associated each tag to its frequency.

\section{Tags assignment}

\section{(a) Estimation of the maximum size of the $3^{\prime} U T R$}

The 3'UTR of a given gene corresponds to the 3' sequence of a mature transcript that is not translated. The size of 3'UTR of Leishmania genes is relatively large and variable. Prior reports estimated, for example, the 3'UTR of HSP70 [32] and HSP83 [33] to 1063 and 886nt, respectively. Moreover, 12201 ESTs of Leishmania available on Genbank were blasted against $L$. major genome. The 800 3'ESTs having a single match and mapping in the 3'UTR of a known gene, with $\geq 80 \%$ identity and over $\geq 50 \%$ of their length, were identified and considered as ESTs with known 3'UTR. Out of these, 129 3'ESTs are L. major ESTs. The size varied from 1 to more than $2000 \mathrm{nt}$; $96 \%$ of the 3'UTRs being less than $1.4 \mathrm{~kb}$ (see Figure 2). This distance will be considered as the maximal tolerated distance between the CDS and the end of the 3'UTR. The list and the description of these 3'UTR is available in Additional file 6. $1.4 \mathrm{~kb}$ is used in the assignment approach paragraph as maximum length of a 3'UTR.

\section{(b) Assignment approach}

The non-redundant set of 9,530 promastigote SAGEtags were mapped to L. major genome by blasting [34] the $14 \mathrm{nt}$ tag sequences against the latest release of the parasite genome available and downloaded from GeneDB [35] on December 8, 2006. The distances between matching tags $(100 \%$ of identity over $100 \%$ of the length) and the STOP codon of the nearest gene were retrieved.

Assigning SMT to the closest predicted CDS is quite natural. The closest CDS being the one located less than $1.4 \mathrm{~kb}$ (maximal tolerated distance between the CDS and the end of the 3'UTR), each SMT found within the coding sequence or $1.4 \mathrm{~kb}$ downstream the STOP codon of a given gene, was assigned to that particular gene. Accordingly, they were classified as SMAT. Tags not verifying these conditions (i.e. SMT within more than $1.4 \mathrm{~kb}$ away from any gene or MMT) were kept for further investigations.

A Gaussian Kernel Density Estimation approach [14] was then used to estimate the density of SMAT distances; the estimation function being:

$$
\hat{f}_{h}(x)=\frac{1}{N h} \sum_{i=1}^{N} K\left(\frac{x-x_{i}}{h}\right)
$$

where $\mathrm{N}$ is the size of SMAT dataset, $\mathrm{x}$ is the set of breakpoints, $\mathrm{h}$ is the bandwidth (smoothing parameter), and $\mathrm{K}$ is the standard Gaussian function with mean zero and variance 1 :

$$
K(x)=\frac{1}{\sqrt{2 p}} e^{-\frac{1}{2} x^{2}}
$$

The X-axis was later split into 50 classes, $\left(x_{i}\right)_{i \leq 50}$, each one corresponding to $41 \mathrm{nt}(41=(\max$ of $\mathrm{x}-\min$ of $\mathrm{x}) /$ 50), and a probability $P_{j}$ was affected to each class $j$. 
$P_{j}$ being equal to the difference between two consecutive Cumulative Density Functions (CDF):

$$
P_{j}=C D F[j+1]-C D F[j] \quad(j=1, \ldots, 49)
$$

where

$$
C D F(j v)=\int_{x}^{x_{j}} \hat{f}_{h}(t) d t
$$

A plot of this probability is shown in Figure 3. To assess the most probable tag-to-gene association, these probabilities were attributed to the distances between the mappings of the MMT and the STOP of the nearest gene. The tag-to-gene association having a probability higher than $5 \%$, were selected.

(c) Differentiate assigned 'sense' and 'antisense' tags SAGE tags being directionally reliable short cDNA sequences [36], we have defined 'sense tags' as tags mapping to a given gene and 'antisense tags' as tags mapping to the reverse complement of a given gene.

\section{Functional characterization of differentially expressed genes}

The 697 tags listed by Guerfali and colleagues [3] (Additional file 7), present in the MDM + Lm and Lm and absent in other human libraries, and differentially expressed, were reloaded. Of these tags, 420 were found

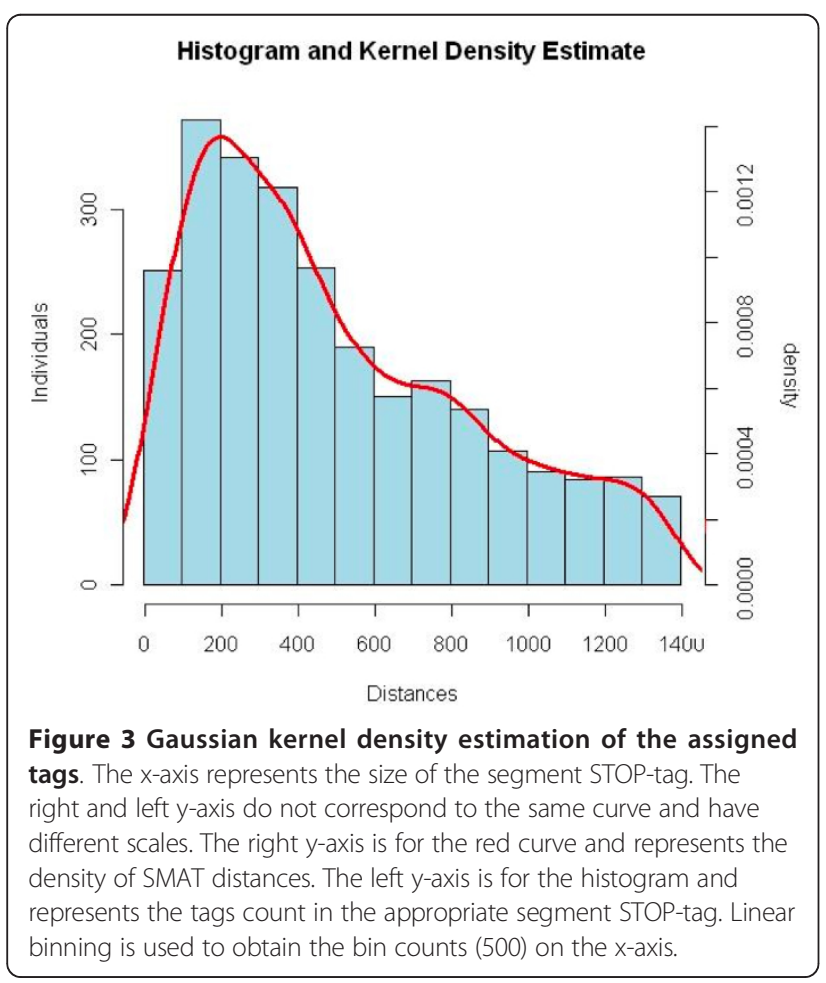

to be preferentially expressed by amastigotes, and 277 by promastigotes. Genes associated to these tags were considered as differentially expressed.

The biological process(es) to which the differentially expressed genes belong to were characterized using GOTermMapper (http://go.princeton.edu/cgi-bin/ GOTermMapper) and GOTermFinder [37].

\section{3'UTR characterization}

SAGE libraries were generated using polyA + RNA and converted to cDNA. The latter were cleaved with the NlaIII enzyme after the first CATG encountered and the 3 '-terminal cDNA fragment were bound to streptavidincoated beads. After concatemerization, these SAGE tags were sequenced. The sequenced tags should correspond to the first $14 \mathrm{nt}$ fragment containing a CATG at the most 3'end position of the mRNA (unless the mRNA contains a second polyA stretch). Departing from this observation, the true positive PRED-A-TERM polyA predictions [31] of all genes with an assigned tag (3094 genes) should be mapping in the 3'UTR of that gene and between the assigned tag and the following CATG.

As recommended by Smith and colleagues [31], PRED-A-TERM was run on the intergenic sequences of all genes with an assigned tag (3094 genes), plus the last $800 \mathrm{nt}$ of the upstream gene, and the first $800 \mathrm{nt}$ of the downstream gene. All predictions mapping in the 3'UTR of the upstream gene and between the corresponding tag and the following CATG or less than 100 nt away from the end of a mapped EST, were considered as a likely true polyA region prediction.

\section{Additional material}

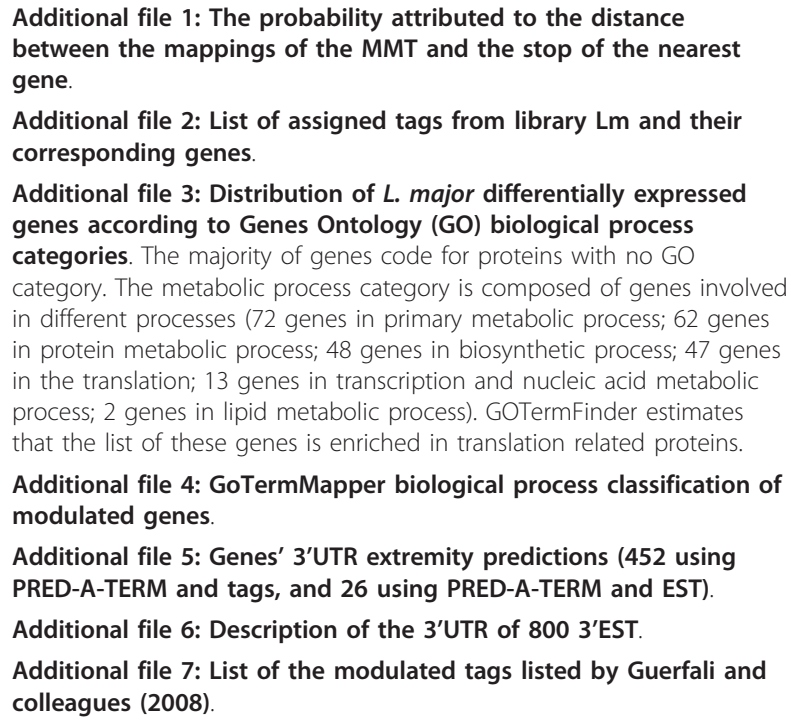




\section{Abbreviations}

SAGE: Serial analysis of gene expression; UTR: UnTranslated Region; CDS: Coding Sequence; DNA: Deoxyribonucleic Acid; CDNA: Complementary Deoxyribonucleic acid; Lm: Promastigotes; MDM + Lm: Leishmania infected Monocyte Derived Macrophages; kde: Kernel Density Estimation; MMT: Multiple Match Tags; SMAT: Single Match Assigned Tags; SMT: Single Match Tags; KEGG: Kyoto Encyclopedia of Genes and Genomes; ORF: Open Reading Frame; EST: Expressed Sequence Tag; mRNA: Messanger Ribonucleic Acid; nt: Nucleotide; Kb: KiloBase; PolyA: PolyAdenosine

\section{Acknowledgements}

We would like to acknowledge Florence Ottones, Laurent Manchon, David Piquemal, Thérèse Commes, and Jacques Marti for participating to the generation of the SAGE libraries published in BMC genomics in 2008 [3]. We would like also to acknowledge Slimane Ben Miled, Karyn Megy, Oliver Hofmann and Abdeljelil Ghram for pre-reviewing this report. This work was done with the support of UNICEF/UNDP/World Bank/WHO Special Programme for Research and Training in Tropical Diseases (the project identifier being A50967) and with the support of the EC Framework Program VII, Project: LEISHDRUG, Grant Agreement No 223414.

\section{Authors' contributions}

Sondos Smandi did all the analyses and participated in the writing Mohamed Farhat did implement the statistical analysis. Alia Benkahla did coordinate all the analyses and the writing. Fatma Guerfali, Dhafer Laouini and Lamia Guizani-Tabbane did participate to the analysis of the results and the writing. Koussay Dellagi did initiate the project and participate to the writing. All authors have read and approved the final manuscript.

\section{Competing interests}

The authors declare that they have no competing interests.

Received: 23 February 2011 Accepted: 27 January 2012

Published: 27 January 2012

\section{References}

1. Ivens AC, Peacock CS, Worthey EA, et al: The genome of the kinetoplastid parasite, Leishmania major. Sci 2005, 309:436-442.

2. Rochette A, Raymond F, Ubeda JM, et al: Genome-wide gene expression profiling analysis of Leishmania major and Leishmania infantum developmental stages reveals substantial differences between the two species. BMC Gen 2008, 9:255.

3. Guerfali FZ, Laouini D, Guizani-Tabbane L, et al: Simultaneous gene expression profiling in human macrophages infected with Leishmania major parasites using SAGE. BMC Geno 2008, 9:238.

4. Srividya G, Duncan R, Sharma P, et al: Transcriptome analysis during the process of in vitro differentiation of Leishmania donovani using genomic microarrays. Parasitol 2007, 134:1527-1539.

5. Saxena A, Lahav T, Holland N, et al: Analysis of the Leishmania donovani transcriptome reveals an ordered progression of transient and permanent changes in gene expression during differentiation. $\mathrm{Mol}$ Biochem Parasitol 2007, 152:53-65.

6. Leifso K, Cohen-Freue G, Dogra N, Murray A, McMaster WR: Genomic and proteomic expression analysis of Leishmania promastigote and amastigote life stages: the Leishmania genome is constitutively expressed. Mol Biochem Parasitol 2007, 152:35-46.

7. Holzer TR, McMaster WR, Forney JD: Expression profiling by wholegenome interspecies microarray hybridization reveals differential gene expression in procyclic promastigotes, lesion-derived amastigotes, and axenic amastigotes in Leishmania mexicana. Mol Biochem Parasitol 2006, 146:198-218

8. McNicoll F, Drummelsmith J, Muller M, et al: A combined proteomic and transcriptomic approach to the study of stage differentiation in Leishmania infantum. Proteomics 2006, 6:3567-3581.

9. Velculescu VE, Zhang L, Vogelstein B, Kinzler KW: Serial analysis of gene expression. Sci (New York, N.Y.) 1995, 270:484-7.

10. Lee $\mathrm{S}$, Clark T, Chen J, et al: Correct identification of genes from serial analysis of gene expression tag sequences. Geno 2002, 79:598-602.

11. Tuteja R, Tuteja N: Serial Analysis of Gene Expression: Applications in Malaria Parasite, Yeast, Plant, and Animal Studies. J Biomed Biotechnol 2004, 2004:106-112.
12. Kim $Y C$, Jung $Y C$, Xuan $Z$, et al: Pan-genome isolation of low abundance transcripts using SAGE tag. FEBS Lett 2006, 580:6721-6729.

13. Boheler KR, Stern MD: The new role of SAGE in gene discovery. Trends Biotechnol 2003, 21:55-58.

14. Sheather SJ, Jones MC: A reliable data-based bandwidth selection method for kernel density estimation. J Royal Statist Soc B 1991, 53:683-690.

15. Kapler GM, Beverley SM: Transcriptional mapping of the amplified region encoding the dihydrofolate reductase-thymidylate synthase of Leishmania major reveals a high density of transcripts, including overlapping and antisense RNAs. Mol Cell Biol 1989, 9:3959-72.

16. Belli Sl, Monnerat $\mathrm{S}$, Schaff $\mathrm{C}$, et al: Sense and antisense transcripts in the histone H1 (HIS-1) locus of Leishmania major. Int J Parasitol 2003, 33:965-75.

17. Monnerat S, Martinez-Calvillo S, Worthey E, et al: Genomic organization and gene expression in a chromosomal region of Leishmania major. $\mathrm{Mol}$ Biochem Parasitol 2004, 134:233-43.

18. Kanehisa M, Goto S, Furumichi M, Tanabe M, Hirakawa M: KEGG for representation and analysis of molecular networks involving diseases and drugs. Nucleic Acids Res 2010, 38:D355-60.

19. Robinson SJ, Cram DJ, Lewis CT, Parkin IA: Maximizing the efficacy of SAGE analysis identifies novel transcripts in Arabidopsis. Plant Physiol 2004, 136:3223-3233.

20. Fizames C, Munos S, Cazettes C, et al: The Arabidopsis root transcriptome by serial analysis of gene expression. Gene identification using the genome sequence. Plant Physiol 2004, 134:67-80.

21. Malig R, Varela C, Agosin E, Melo F: Accurate and unambiguous tag-togene mapping in serial analysis of gene expression. BMC Bioinforma 2006, 7:487.

22. Pleasance ED, Marra MA, Jones SJ: Assessment of SAGE in transcript identification. Genome Res 2003, 13:1203-1215.

23. Cheval L, Pierrat F, Dossat C, et al: Atlas of gene expression in the mouse kidney: new features of glomerular parietal cells. Physiol Geno 2011, 43:161-73.

24. Su W-Y, Xiong H, Fang J-Y: Natural antisense transcripts regulate gene expression in an epigenetic manner. Biochem Biophys Res Commun 2010 396:177-81.

25. Piquemal $\mathrm{D}$, Commes $T$, Manchon $\mathrm{L}$, et al: Transcriptome analysis of monocytic leukemia cell differentiation. Geno 2002, 80:361-371.

26. Welch BL: The generalisation of student's problems when several different population variances are involved. Biom 1947, 34:28-35.

27. Shapiro SS, Wilk MB: An analysis of variance test for normality (complete samples). Biom 1965, 52:591-611.

28. Lu J, Lal A, Merriman B, Nelson S, Riggins G: A comparison of gene expression profiles produced by SAGE, long SAGE, and oligonucleotide chips. Geno 2004, 84:631-6.

29. Clayton C, Shapira M: Post-transcriptional regulation of gene expression in trypanosomes and leishmanias. Mol Biochem Parasitol 2007, 156:93-101.

30. Aline RF, Scholler JK, Stuart K: Transcripts from the co-transposed segment of variant surface glycoprotein genes are in Trypanosoma brucei polyribosomes. Mol Biochem Parasitol 1989, 32:169-78.

31. Smith M, Blanchette M, Papadopoulou B: Improving the prediction of mRNA extremities in the parasitic protozoan Leishmania. BMC Bioinformatics 2008, 9:158.

32. Quijada L, Soto M, Alonso C, Requena JM: Identification of a putative regulatory element in the $3^{\prime}$-untranslated region that controls expression of HSP70 in Leishmania infantum. Mol Biochem Parasitol 2000, 110:79-91.

33. Zilka A, Garlapati S, Dahan E, Yaolsky V, Shapira M: Developmental regulation of heat shock protein 83 in Leishmania. $3^{\prime}$ processing and mRNA stability control transcript abundance, and translation id directed by a determinant in the $3^{\prime}$-untranslated region. J Biol Chem 2001, 276:47922-47929.

34. Altschul SF, Madden TL, Schaffer AA, et al: Gapped BLAST and PSI-BLAST: a new generation of protein database search programs. Nucleic Acids Res 1997, 25:3389-3402.

35. Hertz-Fowler C, Peacock CS, Wood V, et al: GeneDB: a resource for prokaryotic and eukaryotic organisms. Nucleic Acids Res 2004, 32:D339-43.

36. Quéré $R$, Manchon $L$, Lejeune $M$, et al: Mining SAGE data allows largescale, sensitive screening of antisense transcript expression. Nucleic Acids Res 2004, 32:e163. 
37. Boyle El, Weng S, Gollub J, et al: GO::TermFinder-open source software for accessing Gene Ontology information and finding significantly enriched Gene Ontology terms associated with a list of genes. Bioinforma 2004, 20:3710-3715.

doi:10.1186/1756-0500-5-74

Cite this article as: Smandi et al:: Methodology optimizing SAGE library tag-to-gene mapping: application to Leishmania. BMC Research Notes $20125: 74$.

Submit your next manuscript to BioMed Central and take full advantage of:

- Convenient online submission

- Thorough peer review

- No space constraints or color figure charges

- Immediate publication on acceptance

- Inclusion in PubMed, CAS, Scopus and Google Scholar

- Research which is freely available for redistribution

Submit your manuscript at www.biomedcentral.com/submit 